\title{
Chronic gastric impaction and dilatation in horses: Clinical signs, diagnosis, treatment options and pathologic findings - A case series
}

\author{
John Klier', Andreas Blutke², Daniela Emrich², Julia Beckmann', Julia Wittschorek' and Carlos E. Medina-Torres ${ }^{1}$ \\ 1 Centre for Clinical Veterinary Medicine, Equine Clinic, Ludwig-Maximilians-University, Munich, Germany \\ 2 Institute of Veterinary Pathology, Centre for Clinical Veterinary Medicine, Ludwig-Maximilians-University, Munich, Germany
}

\begin{abstract}
Summary: The aim of this study was to summarize and evaluate clinical appearance, laboratory parameters, special ancillary diagnostic tests, treatments and post-mortem pathological findings of chronic gastric impaction and dilatation in horses. Chronic gastric impaction and dilatation is a rare, progressive disease, which is usually detected in its advanced stage. Its aetiology has not yet been fully clarified. Acute primary gastric impaction (from excess volume intake) and secondary gastric impaction (from reflux from the small intestine) must be differentiated from chronic gastric impaction and dilatation. Suspected causes of chronic gastric impaction and dilatation include pyloric stenosis, gastric ulcers, gastric tumours, indigestible feed, feed with high fibre content and dental problems. Motility disorders with resulting gastric impaction and dilatation have also been described in the context of liver diseases. Additional theories suggest damage to the vagus nerve or the intramural nervous system of the stomach and aplasia of the autonomous ganglia of the myenteric and submucosal plexus. It is a retrospective case study of four cases of chronic gastric impaction and dilatation between 2010 and 2013 concerning clinical appearance, laboratory parameters, ancillary diagnostic tests, treatment options and post-mortem pathological findings. For comparison, an additional case of delayed gastric emptying with successful conservative treatment is presented. In one case, nerve degeneration was confirmed and could possibly explain the chronic gastric impaction. A recurrent finding was the adaptive enlargement of the stomach with significant thinning of the muscularis, which was evident in all cases. This is likely to be the result of chronic dilation with a gradually prolonged filling of the stomach leading to 'stretching' of the stomach wall and therefore also a loss of motility, which could be equated to dilated cardiomyopathy, where cardiac output volume decreases with dilation. Gross pathology revealed greatly enlarged stomachs with dry impacted feed material of $43 \mathrm{~kg}, 46 \mathrm{~kg}$ and $108 \mathrm{~kg}$. The treatment options for the disease, whether conservative or surgical depend on the severity and cause of the gastric impaction, and are said to have a very poor or unfavourable prognosis. Chronic gastric impaction can lead to a rupture of the stomach and therefore the prognosis is generally suspected to be grave. Perhaps early identification of delayed gastric emptying and chronic gastric impaction via repetitive gastroscopy could allow for a more successful treatment.
\end{abstract}

Keywords: impaction, dilatation, pathology, stomach, chronic, equine, colic

Citation: Klier J., Blutke A., Emrich D., Beckmann J., Wittschorek J., Medina-Torres C. E. (2017) Chronic gastric impaction and dilatation in horses: Clinical signs, diagnosis, treatment options and pathologic findings. A case series. Pferdeheilkunde 33, 438-446; DOI 10.21836/PEM20170503

Correspondence: Dr. John Klier, Equine Clinic, Centre for Clinical Veterinary Medicine, Ludwig-Maximilians-University Munich, Veterinärstr. 13, 80539 Munich, Germany; e-mail: johnnyklier@hotmail.com

\section{Introduction}

Chronic gastric impaction and dilatation in horses was described by Hutyra and Marek 1922 as a chronic form of impaction which develops over a prolonged time period with a constant adaption of the stomach wall to this condition (Scheidemann and Huthmann 2011, Becker et al. 2017). This can result from primary or secondary causes. Possible primary causes include an overload of the stomach, for example via excessive feed intake or ingestion of feed that can expand, swell or fermt (Müller et al. 1995, Huskamp et al. 2006, Becker et al. 2017). Less frequently, innervation disorders are suspected, which can affect the regular emptying of ingesta as well as its physiological mixing in the stomach to varying degrees (Müller et al. 1995). Secondary gastric impaction can result from reflux caused by small-intestinal ileus or from innervation-related disorders in the intestine. Acute primary (induced by volume overload) and secondary gastric impaction (induced by smallintestinal reflux) must be differentiated from the cases described here, and are not discussed in the present case series.

Chronic gastric impaction and dilatation (CGID) is a rare $(0.3 \%$ incidence according to White and Lessard 1986) progressive disease, which usually only becomes detectable in advanced stages. All age groups can be affected, though it seems to appear more frequently in older horses (Steinberger et al. 2007). Since 1885 seven case reports with size (greater curvature up to $261 \mathrm{~cm}$ ) and weight (up to $122 \mathrm{~kg}$ ) of the stomach have been published (reviewed by Steinberger et al. 2007). More recently, several case reports/series have been published (Parker et al. 2011, Vainio et al. 2011, Scheidemann and Huthmann 2011, Bird et al. 2012, McGovern et al. 2015).

The aetiology of CGID has not yet been fully elucidated. The first description of the disease was provided by Koch (1880) and Fitzroy Phillip (1885) (Müller et al. 1995). The physiological capacity of the equine stomach is given as 8-15 litres (Müller et al. 1995, Nickel et al. 2004). Since regurgitation occurs rarely if ever in horses, due to a very well developed cardiac sphincter (Nickel et al. 2004), an overloading of the stomach can reach its maximum capacity limits quickly. If this occurs slowly over a long period of time, it can result in modification of the stomach with chronic impaction and dilatation (Müller et al. 1995, Huskamp et al. 2006, Becker et al. 2017). If it occurs quickly, however (through reflux or feed overloading) it can result in fatal gastric rupture. Suspected 
causes for CGID are pyloric stenosis (for example through fibrosis as a result of prolonged mural inflammation), decreased motility through neuron degeneration), gastric ulcers, gastric tumours, indigestible feedstuffs, chronic consumption of feed with high fibre content, and dental problems (Müller et al. 1995, Huskamp et al. 2006). Motility disorders with secondary gastric impaction and dilatation have also been reported in association with primary liver disease and hyperammonaemia (McGorum et al. 1999, Gehlen et al. 2010).

Additional theories suggest damage of the vagus and sympathetic nerves as well as the intramural innervation of the stomach, as described in humans (Müller et al. 1995). The disease pattern of aganglionosis (Hirschsprung's Disease) with aplasia of the enteric ganglia of the myenteric and submucosal plexus is also described (Müller et al. 1995). Cases of such aganglionosis, acquired and congenital, are also observed in the horse (Klein et al. 1989, Broekman and Kuiper 2002) and usually lead to adynamic ileus in the gastro-intestinal tract (e.g Lethal White Syndrome). Also plausible are exposure to toxins, as suspected in equine grass sickness in association with Clostridium botulinum type C (Nout 2010c) or oxidative stress due to free radicals, which can lead to nerve damage, as in equine motor neuron disease, equine degenerative myeloencephalopathy and equine pituitary pars intermedia dysfunction (Macleay 2010, Nout 2010a,b; McFarlane and Toribio 2010). However, an exact diagnosis, or proof of the triggering factors is not possible in most cases. Aerophagia, for example in crib-biting, has been identified as a predisposing factor for chronic atony of the stomach (Müller et al. 1995). Additional causes for gastric atony have been described in humans, including anaesthesia and surgery (Müller et al. 1995).

According to Huskamp et al. (2006) and Becker et al. (2017), the disease can progress without clinical signs in its early stages, and develop gradually over months to years. Occasionally, horses may eructate, and a foul smell perceived (Huskamp et al. 2006, Becker et al. 2017). Further clinical signs include bruxism, salivation, weight loss, reduced performance, and occasional postprandial mild colic (Huskamp et al. 2006, Wilson and Blikslager 2011, Becker et al. 2017). Signs of acute gastric overload may also be observed. The left side of the thorax may bulge, as compared to the right side (Huskamp et al. 2006). During per-rectum abdominal palpation it is sometimes possible to feel the barely indentable mass of the stomach, cranial-ventral on the left side (Huskamp et al. 2003 and 2006, Wilson and Blikslager 2011 , Becker et al. 2017).

CGID can persist for years, until a rupture or other cause (emaciation, eating disorder, etc.) leads to euthanasia (Huskamp et al. 2006). Chronic impaction can lead to enlargement of the stomach, after which the risk of rupture is temporarily decreased because of adaption (Huskamp et al. 2006): in such cases, ingesta is thought to be transported over the short path between the cardiac sphincter and pylorus along the lesser curvature (Huskamp et al. 2006, Becker et al. 2017). Chronic gastric impaction mainly affects the body of the stomach (corpus ventriculi), where the saccus caecus tends to hypertrophy and present macroscopically as a double-chambered stomach (Huskamp et al. 2006, Becker et al. 2017). Necropsy reveals frequent adhesions of the stomach to the spleen, the diaphragm and the abdominal wall, secon- dary to chronic ulceration of the gastric mucosa along the margo plicatus (Huskamp et al. 2006, Becker et al. 2017).

The approach to treatment of gastric impaction and dilatation is targeted towards removal of gastric content and prevention of gastric rupture. It requires careful decompression through regular flushing of the stomach with water via nasogastric intubation in order to dissolve or irrigate solid ingesta during sustained fasting (Edwards 2003, Huskamp et al. 2006, Bekker et al. 2017).

Four cases of CGID are presented and discussed with regard to clinical presentation, laboratory findings, additional ancillary diagnostic test results, treatment and post-mortem pathological findings. This retrospective descriptive study includes cases presented to the Equine Hospital, Ludwig-MaximiliansUniversity (LMU) Munich between 2010 and 2013.

\section{Cases reports}

Case 1

A 15-year-old Standardbred mare was presented with recurrent colic signs and emaciation. The mare showed sialorrhoea, bruxism and mild oedema over the ventral thorax. The body condition score was 3 out of 9 (www.aaep.org 2005). As an incidental finding, a grade 2/6 left sided diastolic heart murmur was detected, but heart rate was normal and the rhythm regular. Vital signs were within reference range.

Results of a complete blood count (CBC) and serum chemistry showed physiological values. Faecal flotation examination revealed a mild small strongyle burden. Enteral glucose absorption was slightly reduced (partial malabsorption).

After a fasting period of 14 hours gastroscopic examination revealed accumulation of fibrous ingesta at the entrance of the stomach (cardia). Despite prolonged fasting for an additional $48 \mathrm{~h}$, as well as attempted dissolution of the impacted material with Diet Coca-Cola ${ }^{\mathrm{TM}}$ (not evidence based) via a nasogastric tube and repeated stomach lavage with copious amounts of water, it was not possible to either see into the stomach or reduce the feed content, nor was it possible to pass the gastroscope beyond the food blockage. During per-rectum examination an abnormally distended stomach was not palpable.

Transabdominal ultrasound examination revealed dilatation of the stomach over twelve intercostal spaces (from the 5th to the 16th intercostal space), with the spleen displaced caudally over the entire paralumbar fossa and ventrally across midline, $10 \mathrm{~cm}$ into the right hemi-abdomen. Because of lack of success of the attempted treatment and an unfavourable prognosis, the mare was euthanatized.

\section{Post-mortem pathological findings}

The stomach showed severe dilatation with dimensions of $60 \times 60 \times 30 \mathrm{~cm}$, was filled with $43 \mathrm{~kg}$ of dried ingesta and had multiple chronic ulcers of up to $5 \times 4 \mathrm{~cm}^{2}$ along the margo plicatus and covering approximately 30 percent of the surface of the squamous region. In some of the deeper ulcers 
evidence of bacterial colonies (some with Splendore-Hoeppli Material) could be observed. Multiple adhesions of the omentum were identified and there was no evidence of pyloric stenosis. Microscopically, a generalised, irregular mineralisation of the lamina muscularis, with severe subserosal inflammation was present. The vagus nerve exhibited isolated loss of fibres, but the neurons of the mesenteric ganglia as well as of the myenteric and submucosal displayed microscopically normal. Numbers were not counted. The muscle layers were thin but further investigations were not done. The liver displayed severe bile duct proliferation and changes compatible with a pre-cirrhotic state.

\section{Case 2}

A 14-year-old warmblood gelding was presented with chronic, intermittent, foul-smelling eructation and emaciation. The history described intermittent burping (fermented), fussy eating, increased recumbency after eating and a gradual decrease in performance and progressive weight loss over the last six months. Upon admission to the hospital, the general condition was undisturbed, all vital parameters were within normal range, and body condition score was 2 out of 9 .

Blood analyses (CBC and serum chemistry) and examination of the oral cavity revealed no abnormalities. Faeces consisted of normally digested material and had a normal odour. Gastroscopy after 14 hours of fasting revealed a large accumulation of solid food matter visible at the level of the cardiac sphincter. Oesophageal contractility was normal and the mucosa had no visible abnormalities. Despite three consecutive days of fasting, and attempts of flushing with water and paraffin oil via nasogastric tube, it was not possible to reduce the ingesta or pass the gastroscope beyond the food blockage to see into the stomach. Radiographic images with barium contrast showed no conspicuous features up until the cardia. On rectal examination a markedly displaced spleen was palpable directly in front of the pelvis. Cranial and medial to the spleen, a solid, somewhat indentable and slightly compressible mass could be felt. Ultrasonographic examination of the left hemi-abdomen revealed dilatation of the stomach from the 7th to the 14th intercostal space, with the spleen displaced on the ventral abdomen over midline far to the right side of the body and caudally to the inguinal region. Due to the unfavourable prognosis, the gelding was euthanatized.

\section{Post-mortem pathological findings}

Gross pathology confirmed a chronic gastric impaction and dilatation with $48 \mathrm{~kg}$ of dried ingesta, as well as multifocal gastric ulcers. The musculature of the stomach wall was considerably thinned and focally absent in some areas upon histopathologic examination. Other than the loss of muscle, which was likely secondary to dilatation, there was no hint towards a plausible aetiology for CGID.

\section{Case 3}

An 11 -year-old Westphalian warmblood gelding was presented with gradual weight loss over the past year, poor feed inta- ke and increasing abdominal circumference over the last month without a reduction in performance (Fig. 1). The gelding was ridden daily. Upon admission, the gelding appeared cachectic with body condition score 2 out of 9 and was severely debilitated (Fig. 1). Except for a grade 4/6 left systolic heart murmur, all other clinical examination findings were within normal limits. No oedema was present. Echocardiographic examination revealed a mild mitral valve insufficiency. Blood analyses (CBC and serum chemistry) revealed a moderate to severe hyperproteinaemia $(80 \mathrm{~g} / \mathrm{l})$ with mild hypoalbuminaemia $(27 \mathrm{~g} / \mathrm{l})$, and leucocytes of $12 \times 10^{9} / \mathrm{l}$. Protein electrophoresis showed an hyperglobulinaemia $(\alpha-, \beta-$, and $\gamma$ globulin fraction increased), suggesting a chronic-active inflammatory process. On rectal examination revealed a solid and slightly indentable and compressible mass compatible with the stomach, located deep cranial-ventrally. On abdominal ultrasonography the stomach extended over ten intercostal spaces (from the 8th to 17th intercostal space) on the left side (Fig. 1, 2). The spleen was displaced caudally, ventrally and into the right hemi-abdomen. Gastroscopic examination after 14 hours of fasting demonstrated accumulation of solid food matter occupying the entire stomach (visible at the cardiac sphincter). Despite two additional days of fasting, it was not possible to advance the endoscope further, evaluate the

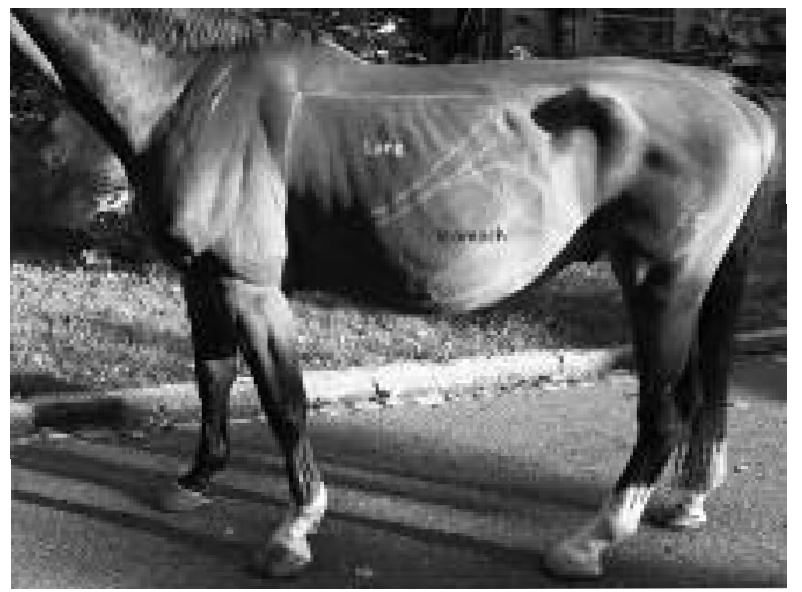

Fig. 1 Chronic gastric impaction and dilatation (case 3), the extent of which is demarcated with chalk on the left ventral abdominal quadrant after ultrasound examination. The ventral lung margin, as determined by percussion is also shown.

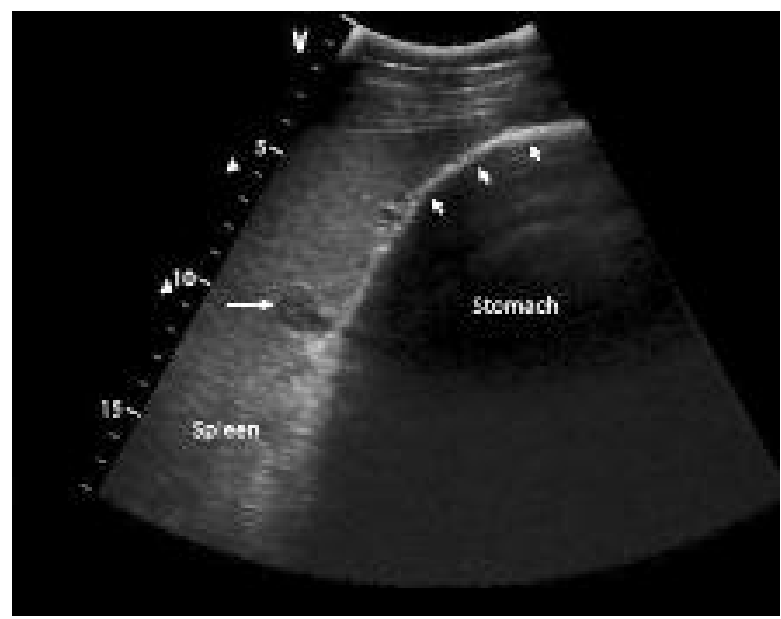

Fig. 2 Ultrasonographic image of the stomach of case 3 (crescent-shaped, hyperechogenic line - arrow heads). A partion of the hilar region of the spleen is also visible (solid arrow). 


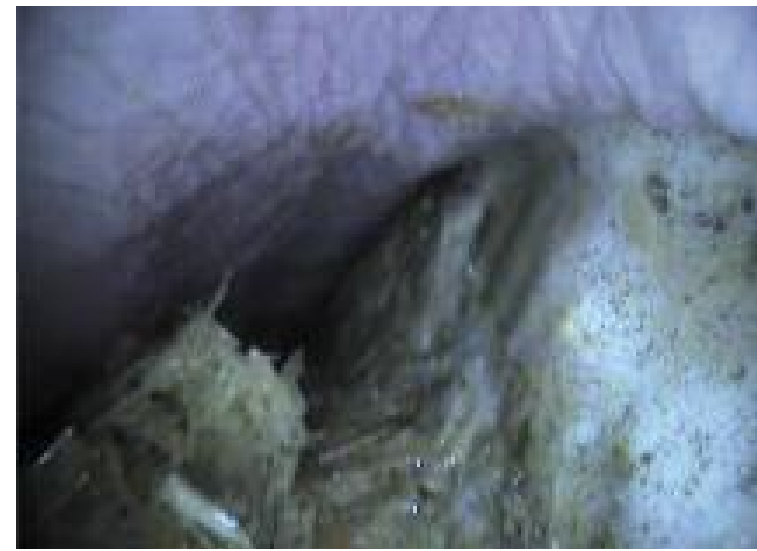

Fig. 3 Endoscopic image of the cardiac region of the stomach of case 3 after 36 hours of fasting demonstrating severe gastric filling. stomach or reduce the food content (Fig. 3). Ventral oedema gradually developed during hospitalisation. Because of the unfavourable prognosis, the horse was euthanatized.

\section{Post-mortem pathological findings}

The stomach was greatly enlarged and contained $108 \mathrm{~kg}$ of dry impacted feed material (Fig. 4A,B,C). Multiple gastric ulcers were present (Fig. 4D, Fig. 5), some of them fully perforating the stomach wall and resulting in a $10 \times 20 \mathrm{~cm}$ adhesion between the stomach and the abdominal wall (Fig. 4A). The musculature of the stomach was severely thinned and partially unrecognizable macroscopically. Histopathological examination confirmed multifocal ulcerative gastritis and hyperkeratosis of the squamous portion, multifocal inflamma-

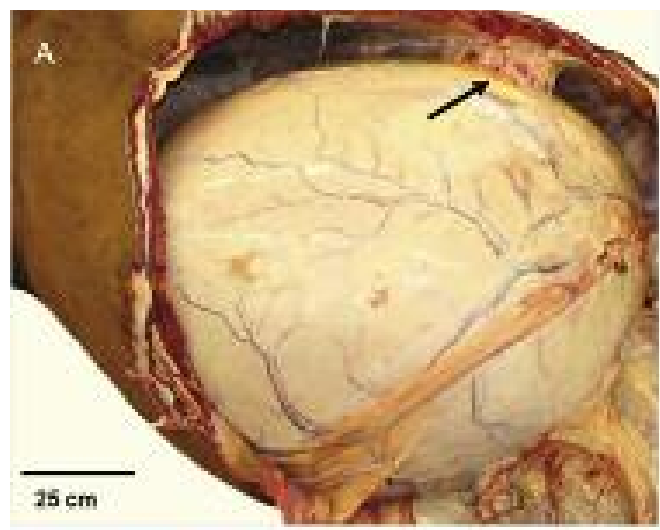

B

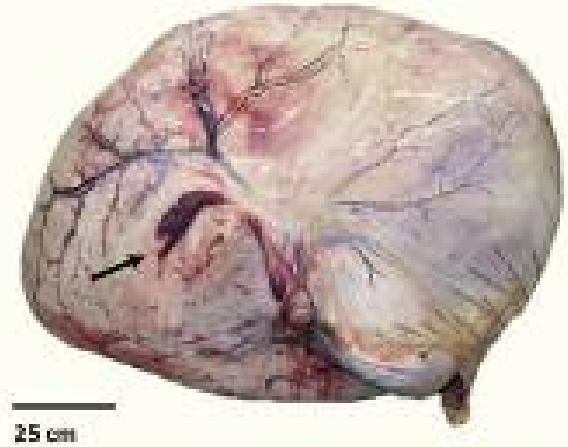

$25 \mathrm{cti}$

Fig. 4 Necropsy finding in case 3 showing a severely distended stomach $(A, B)$ adhered to the abdominal wall following full thickness gastric ulceration (arrow). The stomach contained $108 \mathrm{~kg}$ dry impacted ingesta (C). Multiple chronic gastric ulcers were visible in the nonglandular region of the stomach (D).
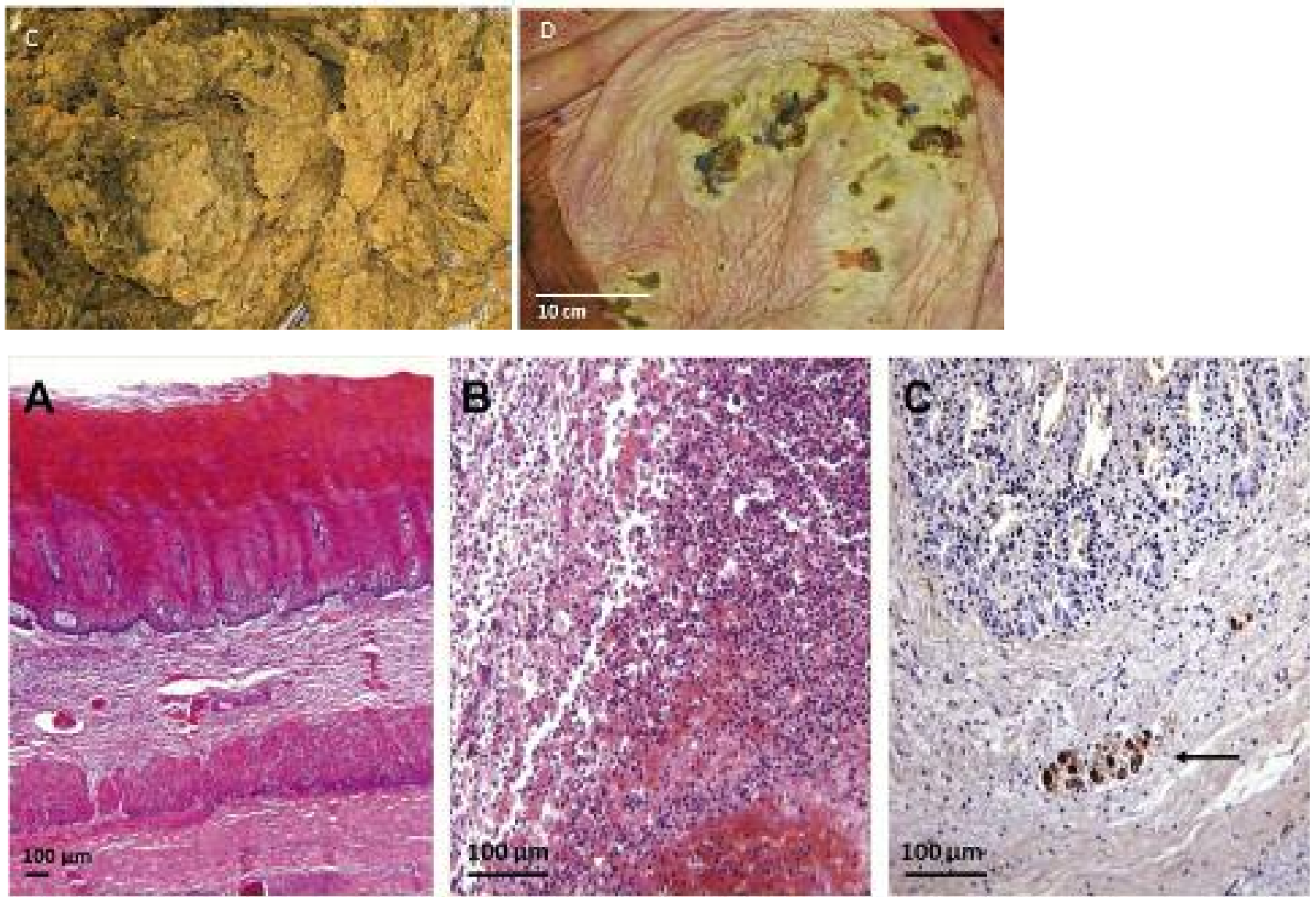

Fig. 5 Hematoxylin-eosin histopathological sections of the stomach wall of case 3 showing marked hyperkeratosis in the non-glandular region of the stomach (A), purulent inflammatory infiltration and severe granulation over the stomach wall (B), and immunohistochemical (antiNSE) demonstration of neuron specific enolase in ganglia of the ileum (arrow) (C). 
tory infiltrates in the gastric mucosa and multifocal fibrosis of the gastric wall. The intramural ganglia of the stomach and the intestine did not display any alterations microscopically.

\section{Case 4}

A 19 year old Lewitzer gelding with a history of recurrent colic episodes over three years was presented. These were usually not associated with abnormalities on transrectal examination except for occasional mild caecal meteorism, and an increasing amount of soluble food material upon nasogastric intubation in the more recent episodes.

At admission, the horse was quiet and in moderate body condition (4-5/9). All vital parameters were within normal range, except for a mild tachypnea. Abdominal auscultation and transrectal abdominal palpation revealed no abnormalities. Abdominal ultrasound revealed severe gastric dilatation to the 16th intercostal space. A gastroscopy after 24 hours of fasting revealed a severely impacted stomach. As treatment, the stomach was flushed three times with 25 litres of water. Upon control gastroscopy after 36 additional hours of fasting the stomach remained severely impacted with crude fibrous material. Because of the unfavourable prognosis, the gelding was euthanized.

\section{Post-mortem pathological findings}

Gross pathology revealed multiple deep gastric ulcers, and partial gastric rupture with adhesions to the spleen. A definitive underlying cause for the chronic gastric impaction could not be identified grossly or on histopathology.

\section{A case of delayed gastric emptying and successful treatment}

A 14-year-old trotter gelding was presented with a fourmonth history of weight loss and muscle wasting. The appetite and food intake remained normal. The teeth had been regularly controlled and faeces were assessed as physiological in consistence. Intestinal parasitism had been ruled out and the horse was under a regular deworming program. Serum chemistry was reported to be within normal range.

Upon examination all vital parameters were within the normal range. The horse showed base narrow stance and muscle fasciculation was evident. The CBC and serum chemistry findings, as well as a protein electrophoresis, were within normal range. An abdominal ultrasound revealed a severely distended stomach from the 8th to the 16th intercostal space. Transrectal examination revealed a caudally displaced spleen with a rounded caudal margin. A compact mass compatible with the stomach could be palpated cranial-medial to the spleen. A peritoneal fluid sample obtained by abdominocentesis was normal. A gastroscopy after 14 hours of fasting revealed a large food impaction in the stomach. Cola was administrated twice ( $3 \mathrm{~L}$ and $2 \mathrm{~L}$ ) via nasogastric intubation and a second and third gastroscopy were performed after additional 18 and 21 hours of sustained fasting. Delayed gastric emptying was diagnosed. After a total of 36 hours of fasting, the stomach could completely explored including the pylorus. No tumour, mass, or functional or mechanical disturbance could be detected. The pylorus demonstrated normal annular peristaltic contractions with phases of opening and closure. The horse was discharged with no report of reoccurrence of the problem after two-year follow-up (by telephone call).

\section{Discussion}

In the cases presented here no definitive aetiology for CGID could be determined, despite extensive clinical, paraclinical and detailed post-mortem examinations. Only in case 1 a denervation-related disorder could be identified as mild histopathological evidence of individual vagal nerve fibre loss in association with inflammatory changes, which could explain the gastric emptying and gastric contractility disorder. However, though the local inflammatory reaction could be possibly due to the presence of bacterial infection, a primary cause for primary gastric inflammation could not be established. Whether the recurrent gastric ulcers detected were of primary or secondary occurrence after chronic gastric impaction and dilatation cannot be determined. They are suspected to be a secondary occurrence, rather than the cause of the gastric motility disorder. An interesting finding was the adaptive enlargement of the stomach with significant thinning of the muscularis, which was evident in all cases. This is likely to be the result of chronic dilation with a gradually prolonged filling of the stomach leading to 'stretching' of the stomach wall and therefore also a loss of motility, which could be equated to dilated cardiomyopathy, where cardiac output volume decreases with dilation. This is in contrast to findings of wall thickening in the cases reported by Bird et al. (2012). These differences could be because of a more chronic and severe stage of disease in the cases reported in this study or because of a different entity. There are further differences between the chronic cases described in this study to more acute cases with a median history of three days (Vainio et al. 2011) in the recent reports: these cases are often presented with overweight and a great appetite (Rodriguez Hurtago et al. 2007 reviewed by Freeman 2011), most reports included ponies (Owen et al. 1987, Rodriguez Hurtago et al. 2007 reviewed by Freeman 2011 ), the presenting complaint was acute colic, and there was less common a prolonged history of sings (Bird et al. 2012). The intestinal motility was usual markedly decreased or absent (Freeman 2011) and the prognosis was usually good with a short term survival of $90 \%$ and a long term survival of $75 \%$ (Vainio et al. 2011). Vainio et al. (2011) reported that four of their 20 cases had an underlying inflammatory bowel disease which could cause decreased gastrointestinal motility and therefore could cause secondary gastric impaction. Also equine dysautonomia could not be ruled out in three cases (Vainio et al. 2011). Ileum biopsies were not performed in these cases. Severe dental problems were identified in $20 \%$ of the cases reported by Vainio et al. (2011). In contrast to the seven cases reported by McGovern et al. (2015) with gastric impaction and concurrent large colon volvulus and a presenting complaint of acute colic without chronic history or weight loss none of the cases reported in this study showed large colon involvement despite markedly enlarged stomachs.

In cases 1 and 3 full thickness ulceration with gastric rupture was observed, resulting in adhesions to the omentum and 
body wall. These adhesions apparently sealed the rupture sufficiently so that it did not result in fatal peritonitis. Aganglionosis could not be detected in any of the cases, and no tumorous changes, strictures, gastric parasites (Gasterophilus spp.) or migrating parasite larvae (Strongylus spp.) were detected in the stomach or the proximal small intestine (Parascaris equorum). An association between CGID and the severe bile duct proliferation and a pre-cirrhotic liver observed in case 1 could not be established. However, it could resemble the secondary chronic gastric impaction and dilatation described in humans with liver cirrhosis (Rost et al. 1938). Although, it was not possible to directly correlate both findings in post mortem examination, a link between the two cannot be absolutely excluded, and warrants future investigation.

Upon necropsy of all cases examined, the stomach contents were found to be very dry, compacted and partially fermented. Dissolution and removal of the stomach contents through irrigation via nasogastric tubing was not possible in any of the cases. Passing the stomach tube into the stomach during the first clinical examination in severe cases of chronic impaction and dilatation can give the impression that the tube passes the cardia but behind it there is no further pushing forward possible if any few centimeters under "pressure" because of resistance of the dry and firm ingesta. (Huskamp et al. 2006, Bekker et al. 2017). This can be used as a further clinical hint.

All horses presented with similar clinical signs including frequent eructation, poor body condition with gradual weight loss, and recurrent mild colic episodes. The differential diagnoses should include a number of other potential causes, such as equine gastric ulcer syndrome (EGUS), tumours (e.g. alimentary lymphoma, gastric squamous cell carcinoma), intestinal malabsorption, chronic caecal and large-intestinal impactions, among others. Clinicopathological findings were unremarkable for all patients, except case 3. However, changes identified in this case were nonspecific and most likely secondary to the chronic disease process, rather than an indication of its cause: i.e. the mild anaemia, hypoalbuminaemia and hyperglobulinaemia were likely associated with chronic inflammation, with maldigestion contributing to hypoalbuminaemia due to protein malnutrition secondary to gastric retention of the ingesta. The cardiac valvular insufficiencies detected in cases 1 and 3 were considered incidental findings, and could not be relate to CGID ante- nor post-mortem.

In the diagnostic assessment of these cases, transabdominal ultrasonography was a simple and fast method for determining the degree of gastric dilatation. In the normal adult horse, the stomach can be easily identified on the left side of the abdomen, as a highly echoic, crescent-shaped, partially double-lined structure medial to the spleen (Fig. 2) and ventral to the lungs. The acoustic window lies between the 8th and the 15th (only the 13th according to others) intercostal space, whereby the stomach should not cover more than five intercostal spaces (Scharner 2011, Cavalleri et al. 2013). To be differentiated from this structure are the large, palisade-shaped pouches of the colon. When extremely filled with gas, fluid or ingesta, it may be enlarged. However, reliable diagnosis of excessive gastric filling is only achieved via nasogastric intubation for decompression of gas and fluid or via repeated gastroscopic examinations after prolonged fasting in cases of retention of ingesta.
The approach to treatment of gastric impaction and dilatation is targeted towards removal of gastric content and prevention of gastric rupture. It requires careful decompression through regular flushing of the stomach with water via nasogastric intubation in order to dissolve or irrigate solid ingesta during sustained fasting. In attempts to improve dissolution of the gastric impaction and achieve complete gastric emptying, a variety of empirical treatments are described. These include administration of Cola drinks, $\mathrm{N}$-acetylcysteine, dioctyl sodium sulfosuccinate (DSS), prokinetics, among others.

It is hypothesised that repeated administration of Cola (e.g. $3 \mathrm{~L}$

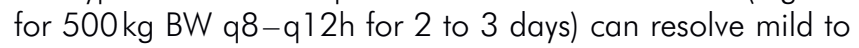
moderate gastric impactions. Dissolution of the impaction is thought to be mediated by the phosphoric (E338) acid present in Cola drinks. However, and despite two previous reports describing its use (Rodriguez Hurtado 2004, Winter et al. 2012), no scientific evidence has been provided to support this therapeutic regimen in horses (May et al. 2012). The use of Cola was adapted from human medicine, where it has been successfully employed in cases of persimmon bezoars (Ladas et al. 2002, Ha et al. 2007). Besides the phosphoric acid, a loosening effect from carbonation is suspected and a possibly more effective dissolution of food matter through $\mathrm{pH}$ reduction and breakage of mucus. A potential side effect of Cola administration in the horse could be laminitis due to the high percentage of eupeptic carbohydrates; however, this has not been described. With caffeine overdose in horses starting at 10-30 $\mathrm{mg} / \mathrm{kg}$ i.v. (Löscher 1999) and about $100 \mathrm{mg}$ caffeine in $1 \mathrm{~L}$ of Cola, side effects due to caffeine are not expected.

In analogy to its application in foals with meconium impactions or the dissolution of chrondroids in the guttural pouches, $\mathrm{N}$-acetylcysteine has been suggested as a further alternative to promote dissolution of impacted gastric ingesta by breaking down the mucus layer that covers the impaction, allowing further water irrigation to penetrate. A dose between $80-100 \mathrm{~g}$ in a $20-40 \%$ solution is suggested. However, no published data exists to support its use in chronic gastric impaction and dilatation. Prokinetic administration could be of benefit as supportive therapy in early and mild cases. However, the use of prokinetics should be reserved for mild cases, as their use in patients with a severely distended stomach and a severe impaction poses the danger of gastric rupture. Metoclopramide $(0.1 \mathrm{mg} / \mathrm{kg}$ in 1 Liter isotonic $\mathrm{NaCl}$ over $60 \mathrm{~min}$ i.v., with higher doses horses can get neurological disorders), a dopamine D2 receptor antagonist, suppresses vagal ganglia and increases acetylcholine release in nerve endings, thus promoting gastrointestinal motility (DiPalma 1990, Okamura et al. 2009). However, it also has side effects on the extrapyramidal system leading to tremors and agitation (Tonin et al. 2004, Okamura et al. 2009). Lidocaine (e.g. $1.3 \mathrm{mg} / \mathrm{kg}$ in $500 \mathrm{ml} \mathrm{NaCl}$ for a $30 \mathrm{~min}$ infusion) also promotes gastrointestinal motility by suppressing local sympathetic nerves (Malone et al. 2006, Okamura et al. 2009). However, a recent study showed that lidocaine therapy had no effect on the prevalence of postoperative reflux (Salem et al. 2016). Side effects include fasciculations and collapse (Malone et al. 2006, Okamura et al. 2009). Cisapride $(1 \mathrm{mg} / \mathrm{kg}), \alpha 5$-hydroxytryptophan 4 receptor agonist and $\alpha 5$ hydroxytryptophan 3 receptor antagonist, also promotes gastrointestinal motility by interacting with its receptor in the myenteric plexus and increases acetylcholine release in nerve 
endings (Fink et al. 2006, Koenig and Cote 2006, Okamura et al. 2009). In humans, cisapride has been reported to cause possible adverse drug reactions like ventricular tachyarrhythmia because of inhibitory effects on $\mathrm{K}^{+}$channels in the heart (Cubeddu 2003, Lillich et al. 2003, Okamura et al. 2009). Cisapride and its sister drug mosapride are currently not available in Germany.

Regardless of treatment, conservative medical management in chronic cases appears to carry an unfavourable prognosis, which likely worsens with increased severity and chronicity of the dilatation (Edwards 2003). Gastric impactions weighing up to $122 \mathrm{~kg}$ have been reported by Huskamp et al. (2000) and Becker et al. (2017). A surgical technique describing gastric massage via laparotomy, combined with lavage via nasogastric intubation and retrograde gastric emptying or else via gastrostomy has been reported (Huskamp et al. 2006). To reduce the size of the dilated stomach, a seromuscular suture in multiple layers from the outside inwards can be performed without resection along the greater curvature (Huskamp et al. 2006, Becker et al. 2017). Because this surgical approach has seldom been performed, data regarding its prognosis is unavailable (Huskamp et al. 2006, Becker et al. 2017). Other sources, however, deem these methods unsuccessful (Wilson and Blikslager 2011). Surgical intervention was not attempted in the cases presented here. Due to the severity, chronicity, advanced stage of debilitation and lack of response to initial treatment, an unfavourable long term prognosis was given to these patients, and euthanasia recommended and elected by the owners. However, it must be acknowledged that reports of successful surgical management of the condition can be found in the veterinary literature, with success apparently depending on the primary cause of the dilatation (Jones et al. 1972, Owen et al. 1987, Parker et al. 2011).

In conclusion, CGID represents a rare disease, and its aetiology and pathogenesis have still not been completely characterised. Furthermore, post-mortem and histopathological examination also often fail to provide a satisfactory explanation. CGID should be considered in the differential diagnosis list of patients with chronic eructation, weight loss, abdominal dilatation, displaced spleen and other signs present in the cases described herein. Prompt recognition of this condition via repetitive gastroscopy is important as treatment options, whether conservative or surgical are limited and success rate is low especially in chronic cases. Thus, the prognosis for long term survival must be considered poor to grave. Perhaps early identification of CGID and delayed gastric emptying could allow for a more successful treatment, but until now, knowledge about the early stages of disease is limited.

\section{Acknowledgements}

The authors thank Ms Elena Serkin for critical revision of the paper.

\section{Conflict of Interest Statement}

The authors of this paper have no financial or personal relationship with other people or organisations that could inappropriately influence or bias the content of the paper.

\section{References}

American Association of Equine Practitioners (AAEP) (2005) Horse health, Overweight horse: Equine Body Condition Score. http://www.aaep.org/info/horse-health?publication $=864$

Becker M., Scheidemann W., Stadtbäumer G. (2017) Magenüberladung und Magendilatation. In: Brehm W., Gehlen H., Ohnesorge B. and Wehrend A (eds), (begründet von Dietz $O$. und Huskamp B.) Handbuch Pferdepraxis, 4th edition, Enke Verlag, Stuttgart, Germany. 498-502.

Bird A. R., Knowles E. J., Sherlock C. E., Pearson G. R., Mair T. S. (2012) The clinical and pathological features of gastric impaction in twelve horses. Equine Vet. J. Suppl. 43,105-110

Broekman L. E. M., Kuiper D. (2002) Megaoesophagus in the horse. A short review of the literature and 18 own cases. Vet. Quart. 24, 199-202

Cavalleri J. M., Bienert-Zeit A., Feige K. (2013) Examination of horses with acute colic - clinical pathology and diagnostic imaging. Tierärzłl. Prax. G 41, 124-134

Cubeddu L. X. (2003) QT prolongation and fatal arrhythmias: a review of clinical implications and effects of drug. Am. J. Ther. 10, 452-457

DiPalma J. R. (1990) Metoclopramide: a dopamine receptor antagonist: Am. Fam. Physician 41, 919-924

Edwards G. B. (2003) Gastric Pathology, 8th Geneva Congress of Equine Medicine and Surgery, Geneva, 2003, proceedings 43-48

Fink C., Tatar M., Failing K., Hospes R., Kressin M., Klisch K. (2006) Serotonin-conatinig cells in the gastrointestinal tract of newborn foals and adult horses. Anat. Histol. Embryol. 35, 23-27

Freeman D. E. (2011) Gastric impaction. Equine Vet. Educ. 23, 174-176

Gehlen H., May A., Venner M. (2010) Lebererkrankungen beim Pferd. Pferdeheilkunde 26, 668-679

Ha S. S., Lee H. S., Jung M. K., Jeon S. W., Cho C. M., Kim S. K., Choi Y. H. (2007) Acute intestinal obstruction caused by a persimmon phytobezoar after dissolution therapy with Coca-Cola. Korean J. Intern. Med. 22, 300-303

Huskamp B., Scheidemann W., Schusser G. F. (2000) Einige seltene Magen- und Duodenumerkrankungen beim erwachsenen Pferd: Zweiphasige Magenruptur, chronische Magendilatation, Duodenumdilatation mit Hypo- und Aganglionose, Längsachsendrehung des Duodenum. Prakt. Tierarzt 82, 729-736

Huskamp B., Kopf N., Scharner D. (2003) Die rektale und sonographische Untersuchung beim Kolikpferd. Opuscula veterinaria, second edition, WAK Verlag, Gescher, Germany.

Huskamp B., Kopf N., Scheidemann W. (2006) Krankheiten des Magens. In: Dietz O., Huskamp B. (eds.), Handbuch Pferdepraxis. Third edition, Enke Verlag, Stuttgart, Germany. 432-435

Jones D. G., Greatorex J. C., Stockman M. J., Harris C. P. (1972) Gastric impaction in a pony: relief via laparotomy. Equine Vet. J. 4, 98-99

Koenig J., Cote N. (2006) Equine gastrointestinal motility-ileus and pharmacological modification. Can. J. Vet. Res. 64, 551-559

Ladas S. D., Kamberoglou D., Karamanolis G., Vlachogiannakos J., Zouboulis-Vafiadis I. (2013) Systematic review: Coca-Cola can effectively dissolve gastric phytobezoars as a first-line treatment. Aliment. Pharmacol. Ther. 37, 169-173

Lillich J. D., Rakestraw P. C., Roussel A. J., Finley M. R., Ganta S., Freeman L. C. (2003) Expression of the ether-a-go-go (ERG) potassium channel in smooth muscle of the equine gastrointestinal tract and influence on activity of jejunal smooth muscle. Am. J. Vet. Res. 64, 267-272

Löscher W. (1999) Pharmaka mit Wirkung auf das Zentralnervensystem. In: Eds: Löscher W., Ungemach F. R., Kroker R. (eds.). Pharmakotherapie bei Haus- und Nutztieren. Fourth edition, Parey, Berlin, Germany. 67-117

Macleay J. M. (2010) Nutritional problems associated with musculoskeletal disorders. In: Reed S. M., Bayly W. M., Sellon D. C. (eds.). Equine internal medicine. Third edition, Saunders Elsevier, St. Louis, USA. 521

Malone E., Ensink J., Turner T., Wilson J., Andrews F., Keegan K., Lumsden J. (2006) Intravenous continuous infusion of lidocaine for treatment of equine ileus. Vet. Surg. 35, 60-66 
May A., Venner M., Cavicchioli E., Gehlen H. (2012) Magenerkrankungen des Pferdes - Diagnostik und Therapie. Pferdeheilkunde 28, 388-405

McFarlane D., Toribio R. E. (2010) Pituitary pars intermedia dysfunction (Equine cushing's disease). In: Reed S. M., Bayly W. M., SelIon D. C. (eds.). Equine internal medicine. Third edition, Saunders Elsevier, St. Louis, USA. 1262-1270

McGorum B., Murphy D., Love S. (1999) Clinicopathological features of equine primary hepatic disease: a review of 50 cases. Vet. Rec. 145, 134-139

McGovern K. F., Suthers J. M., James F. M., O Meara B. J., Parker R. A., Foote A. K., Bladon B. M. (2015) Gastric impaction associated with displacement and volvulus of the large colon in seven mature horses. Equine Vet. Educ. 27, 453-459

Müller E., Donandt D., Pingen C., Zeitelhack M. (1995) Beitrag zur chronischen primären Magendilatation beim Pferd - Ein Fallbericht. Pferdeheilkunde 11, 101-104

Nickel R., Schummer A., Seiferle E. (2004) Lehrbuch der Anatomie der Haustiere. Bd II. Ninth edition, Paul Parey, Stuttgart, Germany. 196

Nout Y. S. (2010a) Equine degenerative myeloencephalopathy. In: Reed S. M., Bayly W. M., Sellon D. C. (eds.). Equine internal medicine. Third edition, Saunders Elsevier, St. Louis, USA. 606-608

Nout Y. S. (2010b) Equine motor neuron disease. In: Reed S. M., Bayly W. M., Sellon D. C. (eds.). Equine internal medicine. Third edition, Saunders Elsevier, St. Louis, USA. 634-637

Nout Y. S. (2010c) Equine grass sickness (Equine dysautonomia). In: Reed S. M., Bayly W. M., Sellon D. C. (eds.). Equine internal medicine. Third edition, Saunders Elsevier, St. Louis, USA. 642

Okamura K., Sasaki N., Yamada M., Yamada H., Inokuma H. (2009) Effects of mosapride citrate, metoclopramide hydrochloride, lidocaine hydrochloride, and cisapride citrate on equine gastric emptying, small intestinal and caecal motility. Res. Vet. Sci. 86, 302-308

Owen R. A., Jagger D. W., Jagger F. (1987) Two cases of equine primary gastric impaction. Vet. Rec. 121, 102-105

Parker R. A., Barr E. D., Dixon P. M. (2011) Treatment of equine gastric impaction by gastrotomy. Equine Vet. Educ. 23, 169-173
Rodriguez Hurtado I. R., Stewart A., Pellegrini-Masini A. (2007) Successful treatment for a gastric persimmon bezoar in a pony using nasogastric lavage with a carbonated cola soft drink. Equine Vet. Educ. 19, 571-574

Rost F., Kessel F. K., Merke F., Meythaler F., Naegeli T. (1938) Pathologische Physiologie Chirurgischer Erkrankungen: Experimentelle Chirurgie, Verdauungsorgane. Springer Verlag, 273

Salem S. E., Proudman C. J., Archer D. C. (2016) Has intravenous lidocaine improved the outcome in horses following surgical management of small intestinal lesions in a UK hospital population? BMC Vet Res. 12,157

Scharner D. (2011) Abdomen. In: Glatzel P. S. (ed.). Atlas der Ultraschalluntersuchung beim Pferd. Schlütersche, Hannover, Germany. 92-113

Scheidemann W., Huthmann S. (2011) A contribution to diseases of the equine stomach: chronic gastric impaction and dilatation. 4th ECEIM Congress Hannover, 2011 , proceedings, 45-46

Steinberg T., Hamann J., Deppenmeier S. (2007) Sekundäre chronische Magenobstipation durch idiopathische muskuläre lleumhypertrophie bei einem Pferd. Pferdeheilkunde 23, 587-592

Tonin M., Cipollina L., Poluzzi E., Crema F., Corazza G. R., De Ponti F. (2004) Review article: clinical implications of enteric and central D2 receptor blockade by antidopaminergic gastrointestinal prokinetics. Aliment. Pharmacol. Ther. 19, 379-390

Vainio K., Sykes B. W., Blikslager A. T. (2011) Primary gastric impact in horses: A retrospective study of 20 cases (2005-2008). Equine Vet. Educ. 23, 186-190

White N. A., Lessard P. (1986) Risk factors and clinical signs associated with cases of equine colic. In: Proceedings of the Association of American Equine Practioners Conference 637-644

Wilson D. A., Blikslager A. T. (2011) Stomach and spleen. In: Aver J. A., Stick J. A. (eds.). Equine surgery. Fourth edition, Saunders Elsevier, Philadelphia, USA. 391

Winter J. C., Schmitz R. R., Gehlen H. (2012) Therapie der primären Magenüberladung des Pferdes. Pferdeheilkunde 28, 437 439 